\title{
Incidence of hypotension according to the discontinuation order of vasopressors in the management of septic shock: a prospective randomized trial (DOVSS)
}

Kyeongman Jeon ${ }^{1,2+}$, Jae-Uk Song ${ }^{3 \dagger}$, Chi Ryang Chung ${ }^{1}$, Jeong Hoon Yang ${ }^{1,4}$ and Gee Young Suh ${ }^{1,2^{*}}$

\begin{abstract}
Background: Vasopressin (AVP) is commonly added to norepinephrine (NE) to reverse shock in patients with sepsis. However, there are no data to support the appropriate strategy of vasopressor tapering in patients on concomitant NE and AVP who are recovering from septic shock. Therefore, the objective of this study was to evaluate the incidence of hypotension while tapering vasopressors in patients on concomitant NE and AVP recovering from septic shock.

Methods: Patients with septic shock receiving concomitant NE and AVP were randomly assigned to taper NE first (NE group) or AVP first (AVP group). The primary end point was the incidence of hypotension within one hour of tapering of the first vasopressor. We also evaluated the association between serum copeptin levels and the occurrence of hypotension.

Results: The study was stopped early due to a significant difference in the incidence of hypotension after 38 and 40 patients were enrolled in the NE group and the AVP group, respectively. There were 26 patients (68.4\%) in the NE group versus 9 patients (22.5\%) in the AVP group who developed hypotension after tapering the first vasopressor $(p<0.001)$. There was a similar finding during the subsequent tapering of the second vasopressor $(64.5 \%$ in the NE vs $25.0 \%$ in the AVP group, $p=0.020$ ). Finally, NE tapering was significantly associated with hypotension during the study period (hazard ratio, 2.221; 95\% confidence interval, 1.106-4.460; $p=0.025$ ). The serum copeptin level was lower in patients in whom hypotension developed during tapering of AVP than it was in those without hypotension.
\end{abstract}

Conclusions: Tapering NE rather than AVP may be associated with a higher incidence of hypotension in patients recovering from septic shock who are on concomitant NE and AVP. However, further studies with larger sample sizes are required to better determine the appropriate strategy for vasopressor tapering.

Trial registration: ClinicalTrials.gov, NCT01493102. Registered on 15 December 2011.

Keywords: Septic shock, Vasopressin, Norepinephrine, Hypotension, Treatment outcome

\section{Background}

Septic shock is characterized by hypovolemia and decreased vascular resistance, with or without myocardial dysfunction [1]. Therefore, administration of intravenous fluids and catecholamines is critical in patients with septic shock

\footnotetext{
* Correspondence: suhgy@skku.edu

${ }^{\dagger}$ Equal contributors

'Department of Critical Care Medicine, Samsung Medical Center,

Sungkyunkwan University School of Medicine, Seoul, Republic of Korea

${ }^{2}$ Division of Pulmonary and Critical Care Medicine, Department of Medicine, and Critical Care Medicine, Samsung Medical Center, Sungkyunkwan University School of Medicine, 81 Irwon-ro, Gangnam-gu, Seoul 06351, Republic of Korea Full list of author information is available at the end of the article
}

to achieve hemodynamic stability and adequate perfusion to vital organs [2]. However, high doses of norepinephrine (NE) often fail to reverse shock, and vasopressin (AVP) can be added with the intent of either raising the mean arterial pressure (MAP) or decreasing the NE dosage [2]. Vasopressin is effective given its vasoconstrictive action and role in replacing AVP deficiency [3]. Given these characteristics, there is increasing interest in adding AVP early, as an adjunctive agent to NE $[4,5]$.

As soon as a patient's hemodynamic variables have stabilized, vasopressor support is gradually tapered in order to decrease the adverse effects of vasopressors 
[6]. However, clinicians must balance the risks from the potential adverse effects of vasopressors with that of hypotension. Hypotension after discontinuation of NE [7-9] or AVP [9-11] has been reported even after stabilization of septic shock. Such hypotension can cause poor organ perfusion (when the pressure is below an organ's critical perfusion pressure), and subsequent injury [12]. However, there are few studies that address vasopressor tapering after shock stabilization $[9,13]$. Furthermore, the incidence of hypotension after vasopressor tapering is not clearly defined, given the variable study populations and vasopressor titration protocols. In particular, there are no data to support the appropriate strategy of vasopressor tapering when AVP and NE are employed concurrently.

Therefore, we evaluated the incidence of hypotension while tapering vasopressors in patients recovering from septic shock on concomitant norepinephrine (NE) and vasopressin (AVP). We also evaluated the role of serum copeptin in predicting development of hypotension, especially during AVP tapering. AVP tapering was particular important to us because relative AVP deficiency has been hypothesized to contribute to the loss of vascular tone in septic shock [10].

\section{Methods}

The prospective randomized, double-blind, controlled trial on the incidence of hypotension, the Discontinuation Order of Vasopressors in the management of Septic Shock (DOVSS) was conducted at Samsung Medical Center (a 1979-bed, university-affiliated, tertiary referral hospital in Seoul, South Korea) between January 2012 and February 2014. The Institutional Review Board of Samsung Medical Center approved the study protocol. Informed consent was obtained from patients or their legally authorized representative prior to enrollment. This study is registered at ClinicalTrials.gov under the identifier NCT01493102.

All patients who were at least 20 years old, and hospitalized in the medical intensive care unit (ICU) were enrolled if they met all the following inclusion criteria: (1) septic shock with documented site (or strong suspicion) of infection; (2) receiving concomitant NE and AVP infusions; (3) MAP $\geq 65 \mathrm{mmHg}$ for at least $2 \mathrm{~h}$ after reducing NE to $0.3 \mathrm{mcg} / \mathrm{kg} / \mathrm{min}$ while maintaining AVP of $0.03 \mathrm{U} / \mathrm{min}$. Exclusion criteria were as follows: terminally ill patients classified as "do not resuscitate;" patients who were suspected to have AVP deficiency (e.g. hypothalamic-pituitary-adrenal axis dysfunction, empty sella syndrome); patients with acute myocardial infarction, congestive heart failure or acute mesenteric ischemia; and patients treated with vasopressors other than NE and AVP.

\section{Initial resuscitation and hemodynamic management of septic shock}

A specific protocol for the early recognition and management of patients with severe sepsis or septic shock was implemented at our center in 2004 [14]. In order to improve compliance with the initial resuscitation bundle and management of sepsis, we revised, approved, and promoted our early goal-directed therapy (EGDT) protocol with an educational program named "Emergency Approach to Sepsis Treatment (EAST)" in early 2008 [15]. Our EGDT protocol is an adaptation of the protocol reported by Rivers et al. [16]. Fluid resuscitation and hemodynamic monitoring were initiated in patients fulfilling the criteria for severe sepsis or septic shock, with placement of a central venous catheter via the internal jugular or subclavian vein approach for central venous pressure (CVP) and central venous oxygen saturation $\left(\mathrm{ScvO}_{2}\right)$ monitoring. Broad-spectrum antibiotics were administered as soon as possible.

Hemodynamic resuscitation was conducted according to a predetermined treatment plan. First, isotonic crystalloid was administered in boluses to a target CVP $\geq 8 \mathrm{mmHg}$. Second, if systolic blood pressure $\geq 90 \mathrm{mmHg}$ or mean arterial pressure (MAP) $\geq 65 \mathrm{mmHg}$ was not achieved with fluid administration, NE was used as a firstline vasopressor to achieve the desired blood pressure. If the target MAP was still not maintained with adequate fluid resuscitation and NE infusion (at $0.3 \mathrm{mcg} / \mathrm{kg} / \mathrm{min}$ ), a supplementary AVP infusion was started at $0.03 \mathrm{U} / \mathrm{min}$. The NE dose was then increased by $0.02 \mathrm{mcg} / \mathrm{kg} / \mathrm{min}$ every $5 \mathrm{~min}$ to achieve the target MAP. Finally, $\mathrm{ScvO}_{2} \geq 70 \%$ was targeted after CVP and blood pressure goals were met. If $\mathrm{ScvO}_{2}$ was $<70 \%$ and the hematocrit was $<30 \%$, packed red blood cells were transfused to achieve a hematocrit of at least $30 \%$. If the $\mathrm{ScvO}_{2}$ remained $<70 \%$ when hematocrit was $\geq 30 \%$, dobutamine was initiated at the treating physician's discretion and titrated in attempts to reach $\mathrm{ScvO}_{2} \geq 70 \%$. When the patient remained hypotensive after at least one hour of resuscitation with fluids and vasopressors [17], lowdose corticosteroid therapy was recommended as soon as possible after adrenocorticotropic hormone was measured, if possible. However, the time to initiation of low-dose corticosteroid therapy was decided by the treating physician in the emergency department or ICU. Hydrocortisone was administered intravenously every $6 \mathrm{~h}$ as a $50-\mathrm{mg}$ bolus for 5 days, and then tapered (50 $\mathrm{mg}$ intravenously every $12 \mathrm{~h}$ for 3 days, followed by $50 \mathrm{mg}$ intravenously daily for 3 days). Fludrocortisone was not administered in conjunction with hydrocortisone. If hemodynamic stabilization was achieved, the vasopressor was tapered at the discretion of the attending physician, keeping MAP $>65 \mathrm{mmHg}$ and urinary output $>0.5 \mathrm{~mL} / \mathrm{kg} / \mathrm{h}$. 
Vasopressor withdrawal protocol and patient assignment If the target MAP was met and maintained for $2 \mathrm{~h}$ with hemodynamic management, NE was titrated to the dose of $0.3 \mathrm{mcg} / \mathrm{kg} / \mathrm{min}$ by $0.02 \mathrm{mcg} / \mathrm{kg} / \mathrm{min}$ every $5 \mathrm{~min}$, keeping MAP >65 mmHg. If the MAP remained stable at > $65 \mathrm{mmHg}$ for another hour, then written informed consent was obtained from the participants. Participants were then randomly assigned to reduce NE first (NE group) or AVP first (AVP group) after another hour, if the target MAP was maintained. A computer-generated randomization list in blocks of four was used for treatment allocation. The randomization procedure and treatment allocation were performed by the research coordinator. The sequence was concealed from patients and investigators until the interventions were completed.

Vasopressors were only tapered when the MAP had been maintained at $\geq 65 \mathrm{mmHg}$ with a constant infusion of both NE $(0.3 \mathrm{mcg} / \mathrm{kg} / \mathrm{min})$ and AVP $(0.03 \mathrm{U} / \mathrm{min})$, for at least $2 \mathrm{~h}$. In the NE group, NE was discontinued at the rate $0.1 \mathrm{mcg} / \mathrm{kg} / \mathrm{min}$ every hour, keeping the AVP infusion at $0.03 \mathrm{U} / \mathrm{min}$; next, AVP was weaned at a rate $0.01 \mathrm{U} / \mathrm{min}$ every hour if the MAP was maintained above $65 \mathrm{mmHg}$ for $2 \mathrm{~h}$ after successful termination of NE. In the AVP group, AVP was discontinued first and then NE was subsequently withdrawn in the same manner as in the NE group (with the exception of the order). The vasopressor discontinuation continued until the development of hypotension or complete withdrawal of all infused vasopressors. If hypotension developed during vasopressor withdrawal, one or more subsequent interventions were used to maintain the target MAP. These interventions were performed according to the measured CVP. Administration of a fluid challenge (of at least $30 \mathrm{~mL} / \mathrm{kg}$ of IV crystalloid or equivalent volume of colloid over $30 \mathrm{~min}$ ) was initially performed to keep CVP $>8 \mathrm{mmHg}$. If CVP was $\geq 8$, the discontinued vasopressor was increased up to its dose prior to the hypotension, and then increased according to the protocol (NE, $0.1 \mathrm{mcg} / \mathrm{kg} / \mathrm{min}$ and AVP, $0.0 \mathrm{l} \mathrm{unit} / \mathrm{min}$ ) to maintain the target MAP. If the target MAP was not achieved despite these interventions, the NE dose was increased by $0.1 \mathrm{mcg} / \mathrm{kg} / \mathrm{min}$ every hour until the MAP stabilized. If hypotension developed during weaning of the second vasopressor, the interventions were performed in the same way. The discontinued agent could be restarted if AVP was titrated to the maximum dose of $0.03 \mathrm{U} / \mathrm{min}$ or if the required NE infusion exceeded $0.3 \mathrm{mcg} / \mathrm{kg} / \mathrm{min}$. Vasopressors were titrated by the bedside nurse and treating physician based on the described study protocol, to maintain the target MAP (Fig. 1).

\section{Study outcomes}

The primary outcome was the incidence of hypotension within one hour after tapering the first vasopressor, which was defined as a sustained decrease in MAP $<65 \mathrm{mmHg}$ despite adequate fluid resuscitation. Secondary outcomes included the overall incidence of hypotension during the entire study period, the incidence of hypotension according to the tapering of each vasopressor, ICU mortality, 28-day mortality, and hospital mortality. We also evaluated the association between serum copeptin levels and hypotension during AVP tapering to determine if development of hypotension from AVP tapering is associated with AVP deficiency.

\section{Measurement of serum copeptin}

Measurement of circulating AVP is problematic due to its short half-life, instability, and cumbersome detection methods [18]. In contrast, copeptin is a stable fragment that is located at the $\mathrm{C}$ terminal of provasopressin. Copeptin levels directly mirror AVP levels because of its stoichiometric synthesis [19]. Copeptin also exhibits an advantageous biochemical profile for rapid and reliable laboratory testing [19]. Therefore, copeptin has recently been suggested to be a surrogate marker of AVP. Furthermore, one study found that levels of serum copeptin and AVP were strongly correlated in patients with septic shock [20]. Serum copeptin levels were checked in our study using the Copeptin (Human) EIA Kit (CSB-E12130h; CUSABIO CO, Ltd., China), according to the manufacturer's instructions. The results are expressed in picograms per milliliter. The calibration range was $19.5 \mathrm{pg} / \mathrm{mL}$ to $5000 \mathrm{pg} / \mathrm{mL}$, with a detection limit of $19.5 \mathrm{pg} / \mathrm{mL}$. Intra-assay and inter-assay variance was $<8 \%$ and $10 \%$, respectively.

\section{Statistical analysis}

We initially calculated that a sample size of 122 (with 61 per group) was required for enrollment and randomization to detect an absolute difference of $25 \%$ in the incidence of hypotension, with two-sided alpha error of 0.05 and power of $80 \%$. This sample size assumed a $55 \%$ incidence of hypotension after the initial tapering AVP according to previous study [9]. After considering a dropout rate of $10 \%$, we ultimately needed 134 patients. A preplanned interim analysis was scheduled after enrollment of at least 60\% (80 patients) of the planned 134 patients. An O'Brien-Fleming approach was used for sequential stopping rules for safety according to the Lan-DeMets method with an a priori $p$ value of 0.025 for stopping [21]. After interim analysis, the study could be stopped, since there was a significant difference in the incidence of hypotension between the two groups. We applied a modified intention-to-treat principle by only analyzing patients who completely followed the study protocol, as this study was designed to evaluate the effect on the incidence of hypotension according to vasopressor tapering (Fig. 2).

Data were compared using the Mann-Whitney U test for continuous variables and the chi-square or Fisher's 


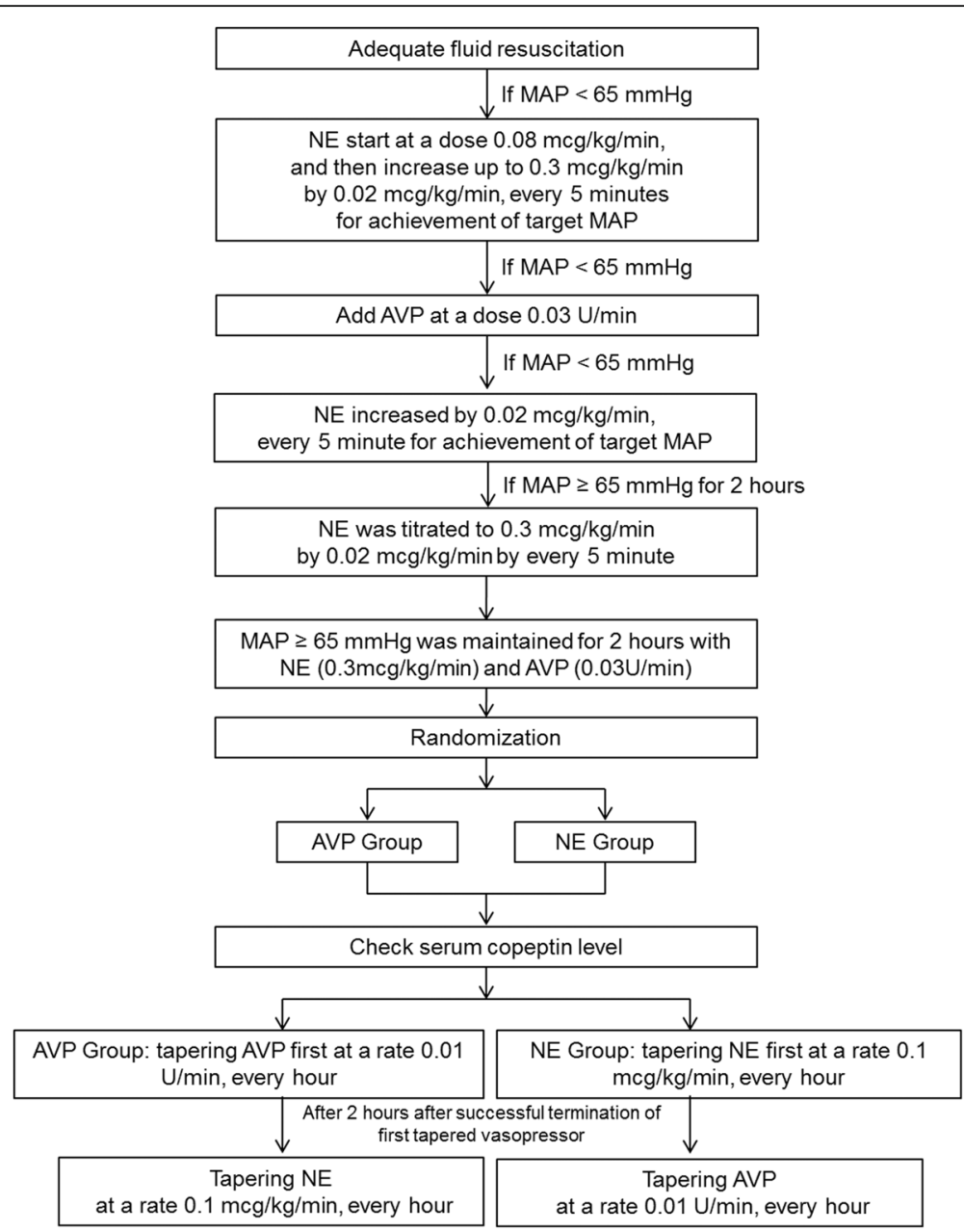

Fig. 1 Study protocol on titrating vasopressors. NE, norepinephrine; MAP, mean arterial pressure; AVP, vasopressin

exact test for categorical variables. Primary analysis, which compared the incidence of hypotension within one hour of tapering the first vasopressor in the two groups, was evaluated using an unadjusted chi-square test. We also compared clinical parameters, including serum copeptin levels, between the two groups who were classified according to development of hypotension and tapered vasopressor in the subgroups with hypotension development. In order to identify the factors associated with development of hypotension after vasopressor tapering, continuous variables were converted to categorical variables using median cutoff values for multivariable analysis. The chi-square or Fisher's exact test was used to assess differences between the dichotomous variables. The Cox regression model was used for time-to-event analysis to assess the overall incidence of hypotension during the study period. Time zero for this analysis was defined as the point of tapering the first vasopressor. The variables with a $p$ value $<0.25$ in univariable analysis were entered into a Cox regression model. All tests were two-sided. A $p$ value $<0.05$ was considered statistically significant.
Data were analyzed using PASW statistical software version 17 (SPSS, Chicago, IL, USA).

\section{Results}

Baseline patient characteristics

A total of 624 patients with septic shock who were admitted to the medical ICU were screened for inclusion. After 539 patients were excluded, 85 patients underwent randomization. Of these patients, two withdrew consent after randomization, and four did not have vasopressors tapered according to the study protocol for various reasons. Additionally, one patient clinically decompensated prior to vasopressor tapering. The preplanned interim analysis was ultimately performed in 78 patients, with 38 in the NE group and 40 in the AVP group (Fig. 2). However, serum copeptin levels were unavailable in two patients because of technical errors. After interim analysis, the study was stopped without protocol modification, because there was a significant difference between the two groups in the incidence of hypotension during the first vasopressor tapering. 


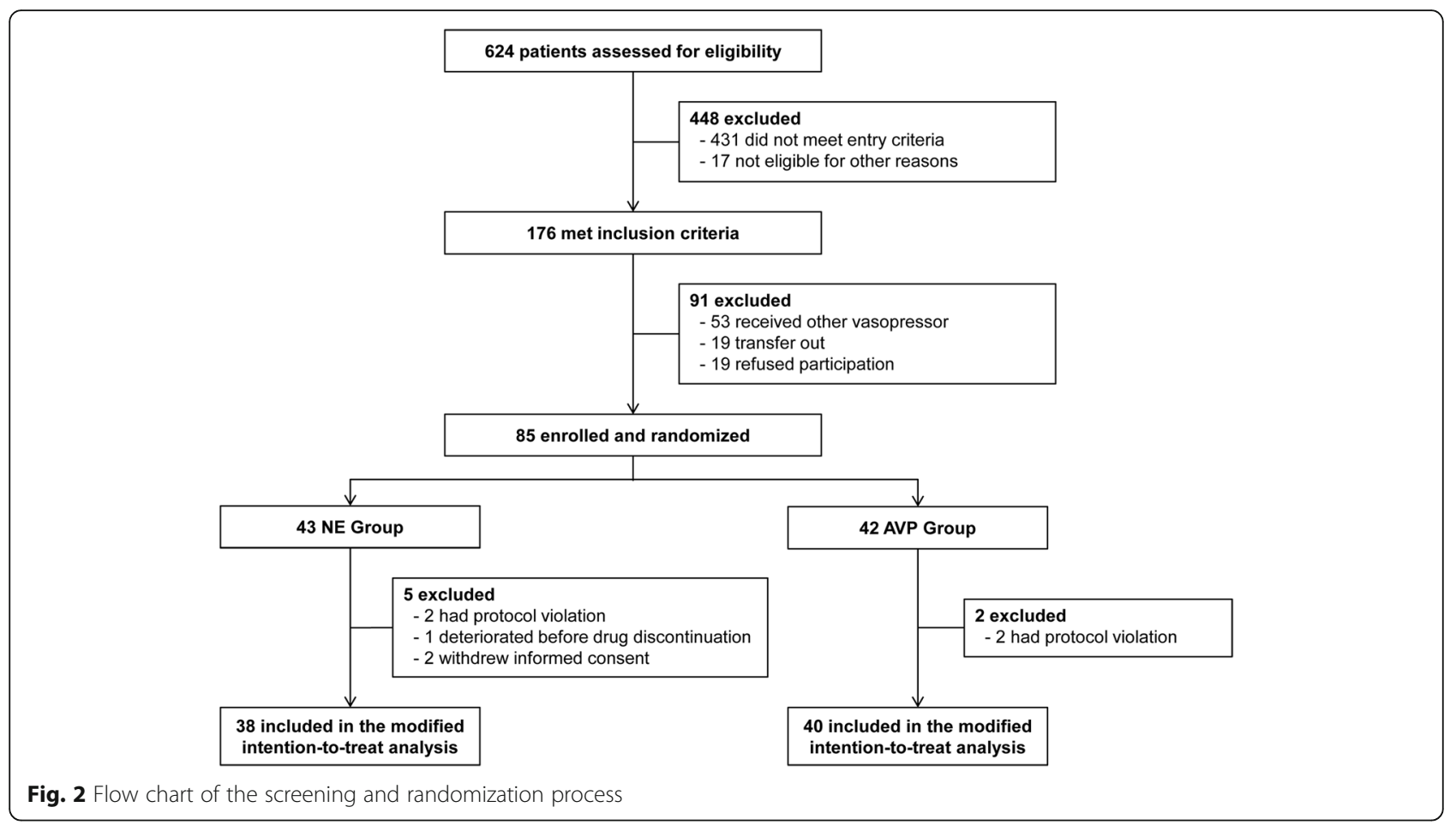

The baseline characteristics of the enrolled patients are summarized in Table 1 . There were 49 men with a median age of 66 years (56-71 years). Pneumonia was the most common cause of septic shock $(n=39,50 \%)$. The median sequential organ failure assessment (SOFA) score and simplified acute physiology score 3 (SAPS3) were 10 (8-12) and 74 (63-84), respectively. The median serum copeptin level was $162 \mathrm{pg} / \mathrm{mL}(107-220)$ at a median time to randomization of $24.2 \mathrm{~h}$, with no statistically significant difference between the two groups $(p=0.640)$. There was no significant difference between groups in MAP or CVP measured during the study period (Table 1). Patients' characteristics were well-balanced between the AVP group and NE group, except for arterial partial pressure of oxygen $(\mathrm{PaO} 2) /$ fraction of inspired oxygen (FiO2) (PF ratio) and need for mechanical ventilation. The patients in the AVP group had lower PF ratios $(p=0.014)$ and received more mechanical ventilation $(p=0.009)$, than did those in the NE group.

\section{Incidence of hypotension during vasopressor tapering}

There were 26 patients (68.4\%) in the NE group versus 9 patients $(22.5 \%)$ in the AVP group who developed hypotension within one hour after tapering the first vasopressor $(p<0.001$, Table 2$)$. There was a similar finding during the subsequent tapering of the second vasopressor $(64.5 \%$ vs $25.0 \%, p=0.020)$. Therefore, $\mathrm{NE}$ tapering was significantly associated with development of hypotension. However, there were no significant differences in the overall incidence of hypotension during the entire study period between the two groups (Table 2). There were $23(57.5 \%)$ and $13(34.2 \%)$ patients who died during hospitalization in the AVP and NE groups, respectively. Hospital mortality was higher in the patients in the AVP group (Table 2).

\section{Serum copeptin levels according to group}

The serum copeptin level was not significantly associated with the order of vasopressor tapering (Fig. 3a). However, the copeptin level was significantly lower in patients who developed hypotension in the AVP group (Fig. 3b), or during the entire experimental period (Fig. 3e). However, these differences were not observed in the NE group (Fig. 3c) or during the entire experimental period (Fig. 3f).

The clinical characteristics were compared between the groups according to which vasopressor was tapered immediately before developing hypotension in the subgroups of patients who developed hypotension (Table 3). The only significant difference was observed in the serum copeptin levels, which were much lower in patients who developed hypotension during AVP tapering than in those who developed hypotension during NE $(p<0.001)$.

\section{Clinical factors associated with hypotension}

Univariable comparisons of clinical variables were performed to identify factors associated with hypotension after vasopressor tapering (Table 4). Interestingly, low serum $\mathrm{C}$-reactive protein (CRP) was associated with the development of hypotension. In addition, following 
Table 1 Baseline characteristics of enrolled patients with septic shock

\begin{tabular}{|c|c|c|c|c|}
\hline & All patients $(N=78)$ & AVP group $(n=40)$ & NE group $(n=38)$ & $P$ value \\
\hline Age, years & $66(56-71)$ & $67(56-73)$ & $64(55-69)$ & 0.206 \\
\hline Gender, male & $49(62.8)$ & $25(62.5)$ & $24(63.2)$ & 0.952 \\
\hline Causes of septic shock ${ }^{\mathrm{a}}$ & & & & 0.522 \\
\hline Pneumonia & $39(50.0)$ & $23(57.5)$ & $16(42.1)$ & \\
\hline Intraabdominal infection & $22(28.2)$ & $10(25.0)$ & $12(31.6)$ & \\
\hline Urogenital infection & $16(20.5)$ & $7(17.5)$ & $9(23.7)$ & \\
\hline Cather related infection & $5(6.4)$ & $4(10.0)$ & $1(2.6)$ & \\
\hline Endocarditis & $2(2.6)$ & $1(2.5)$ & $1(2.6)$ & \\
\hline Others $^{\mathrm{b}}$ & $3(3.8)$ & $1(2.5)$ & $2(5.3)$ & \\
\hline MAP before initial resuscitation, $\mathrm{mmHg}$ & $52(45-57)$ & $52(46-57)$ & $54(44-60)$ & 0.255 \\
\hline CVP before initial resuscitation, $\mathrm{mmHg}$ & $7(4-9$ & $6(4-8)$ & $7(5-9)$ & 0.303 \\
\hline MAP at the time of vasopressor initiated, $\mathrm{mmHg}$ & $55(51-60)$ & $54(50-60)$ & $56(52-60)$ & 0.309 \\
\hline CVP at the time of vasopressor initiated, $\mathrm{mmHg}$ & $11(9-14)$ & $10(8-14)$ & $11(9-13)$ & 0.954 \\
\hline Total bilirubin, mg/dL & $1.05(0.50-2.30)$ & $1.15(0.50-2.38)$ & $0.95(0.50-2.01)$ & 0.802 \\
\hline Serum creatinine, mg/dL & $1.34(0.93-1.99)$ & $1.48(0.84-1.94)$ & $1.30(0.98-2.26)$ & 0.960 \\
\hline Lactic acid, mmol/L & $3.61(2.40-5.44)$ & $3.53(2.41-5.48)$ & $4.11(2.39-5.65)$ & 0.699 \\
\hline Procalcitonin, ng/mL & $12.70(3.29-37.70)$ & $14.35(3.52-45.66)$ & $9.91(2.62-31.42)$ & 0.484 \\
\hline C-reactive protein, $\mathrm{mg} / \mathrm{mL}$ & $13.96(6.87-24.35)$ & $16.04(8.29-26.10)$ & $12.99(6.09-23.17)$ & 0.492 \\
\hline Maximum NE dose during study period, ug/kg/min & $0.68(0.40-1.20)$ & $0.68(0.40-1.45)$ & $0.68(0.40-1.03)$ & 0.195 \\
\hline SAPS3 & $74(63-84)$ & $75(66-92)$ & $72(61-82)$ & 0.192 \\
\hline SOFA score & $10(8-12)$ & $10(8-12)$ & $10(7-11)$ & 0.793 \\
\hline \multicolumn{5}{|l|}{ Clinical status on randomization } \\
\hline MAP, mmHg & $77(71-81)$ & $75(68-81)$ & $77(74-80)$ & 0.237 \\
\hline $\mathrm{CVP}, \mathrm{mmHg}$ & $10(8-14)$ & $10(8-14)$ & $10(8-14)$ & 0.811 \\
\hline Need for mechanical ventilation & $54(69.2)$ & $33(82.5)$ & $21(55.3)$ & 0.009 \\
\hline Need for renal replacement therapy & $22(28.2)$ & $11(27.5)$ & $11(28.9)$ & 0.887 \\
\hline Need for dobutamine & $6(7.7)$ & $1(2.5)$ & $5(13.2)$ & 0.104 \\
\hline SOFA score & $12(10-15)$ & $12(11-15)$ & $12(9-15)$ & 0.413 \\
\hline PF ratio & $164.5(100.2-264.1)$ & $132.4(96.1-202.6)$ & $198.9(133.0-290.7)$ & 0.014 \\
\hline Total bilirubin, mg/dL & $1.30(0.68-3.70)$ & $1.30(0.63-2.93)$ & $1.35(0.65-4.23)$ & 0.845 \\
\hline Serum creatinine, mg/dL & $1.12(0.72-1.95)$ & $1.10(0.75-1.92)$ & $1.23(0.68-1.95)$ & 0.881 \\
\hline Corticosteroid treatment & $72(96.0)$ & $38(97.4)$ & $34(94.4)$ & 0.605 \\
\hline Time to randomization & $24.2(13.1-44.1)$ & $27.4(15.1-44.4)$ & $19.2(11.5-33.1)$ & 0.108 \\
\hline Total vasopressor duration before tapering first vasoactive agent, hours & $24.2(13.1-41.8)$ & $29.0(15.1-43.9)$ & $19.2(11.5-33.1)$ & 0.127 \\
\hline Copeptin, pg/mL $(n=76)$ & $162(107-220)$ & $148(100-237)$ & $170(113-215)$ & 0.640 \\
\hline
\end{tabular}

Data are presented as frequencies (number of patients), with the percentages in parenthesis, or as medians with interquartile ranges (IQR) in parenthesis ${ }^{a}$ More than one criterion can be used

${ }^{\mathrm{b}}$ Others included meningitis $(n=1)$ and deep neck $(n=1)$ and soft tissue infections $(n=1)$

AVP, vasopressin; CVP, central venous pressure; MAP, mean arterial pressure; $N E$, norepinephrine; $P F$ ratio, arterial partial pressure of oxygen ( $\mathrm{PaO} 2) /$ fraction of inspired oxygen (FiO2) ratio; SAPS3, simplified acute physiology score 3; SOFA, sequential organ failure assessment

vasopressor tapering the median serum copeptin level was lower in patients who developed hypotension $(144 \mathrm{pg} / \mathrm{mL}$, IQR $105-199 \mathrm{pg} / \mathrm{mL}$ ) than it was in those who did not (223 pg/mL, IQR 109-281 pg/mL, $p=0.032)$. NE tapering was significantly associated with hypotension $(p=0.035)$. However, there were no significant differences between the two groups with regard to mortality or length of stay. In a multivariable analysis using a Cox proportional hazards model, hypotension was only significantly associated with NE tapering during the entire experimental period (adjusted hazard ratio, 2.221; 95\% confidence interval, $1.106-4.460 ; p=0.025)$. 
Table 2 Outcomes by treatment group

\begin{tabular}{|c|c|c|c|}
\hline & AVP group $(n=40)$ & NE group $(n=38)$ & $P$ value \\
\hline \multicolumn{4}{|l|}{ Development of hypotension within one hour after tapering of vasopressor } \\
\hline Hypotension on tapering the first vasopressor & $9(22.5)$ & $26(68.4)$ & $<0.001$ \\
\hline Hypotension on tapering sequential second vasopressor $(n=43)$ & $20(64.5)$ & $3(25.0)$ & 0.020 \\
\hline Hypotension on tapering the first or second vasopressor & $29(72.5)$ & $29(76.3)$ & 0.700 \\
\hline Time to hypotension after tapering vasopressor, hours $(n=58)$ & $4.3(2.5-5.1)$ & $2.0(1.2-2.5)$ & $<0.001$ \\
\hline MAP at the time of hypotension developed on tapering of vasopressor, $\mathrm{mmHg}(n=58)$ & $61(58-62)$ & $62(59-63)$ & 0.111 \\
\hline CVP at the time of hypotension developed on tapering of vasopressor, $\mathrm{mmHg}(n=58)$ & $10(7-14)$ & $9(6-13)$ & 0.810 \\
\hline Total vasopressor duration, hours & $58.4(33.9-100.0)$ & $43.8(28.9-81.9)$ & 0.169 \\
\hline \multicolumn{4}{|l|}{ Clinical outcomes } \\
\hline ICU mortality & $15(37.5)$ & $11(28.9)$ & 0.423 \\
\hline ICU length of stay, days & $9(6-13)$ & $7(2-12)$ & 0.107 \\
\hline 28-day mortality & $17(42.5)$ & $12(32.4)$ & 0.362 \\
\hline Hospital mortality & $23(57.5)$ & $13(34.2)$ & 0.039 \\
\hline Hospital length of stay, days & $25(15-38)$ & $21(13-37)$ & 0.542 \\
\hline
\end{tabular}

AVP, vasopressin; NE, norepinephrine; MAP, mean arterial pressure; CVP, central venous pressure; ICU, intensive care unit

\section{Discussion}

In this randomized controlled trial on the incidence of hypotension with vasopressor tapering, we found that hypotension developed more commonly in patients in whom NE was tapered first. In a Cox proportional hazards model, NE tapering was significantly associated with hypotension. In patients in whom hypotension developed during AVP tapering, however, the serum copeptin level was significantly lower than it was in those without hypotension.

There are guidelines on vasopressor initiation in the hemodynamic management of patients with septic shock

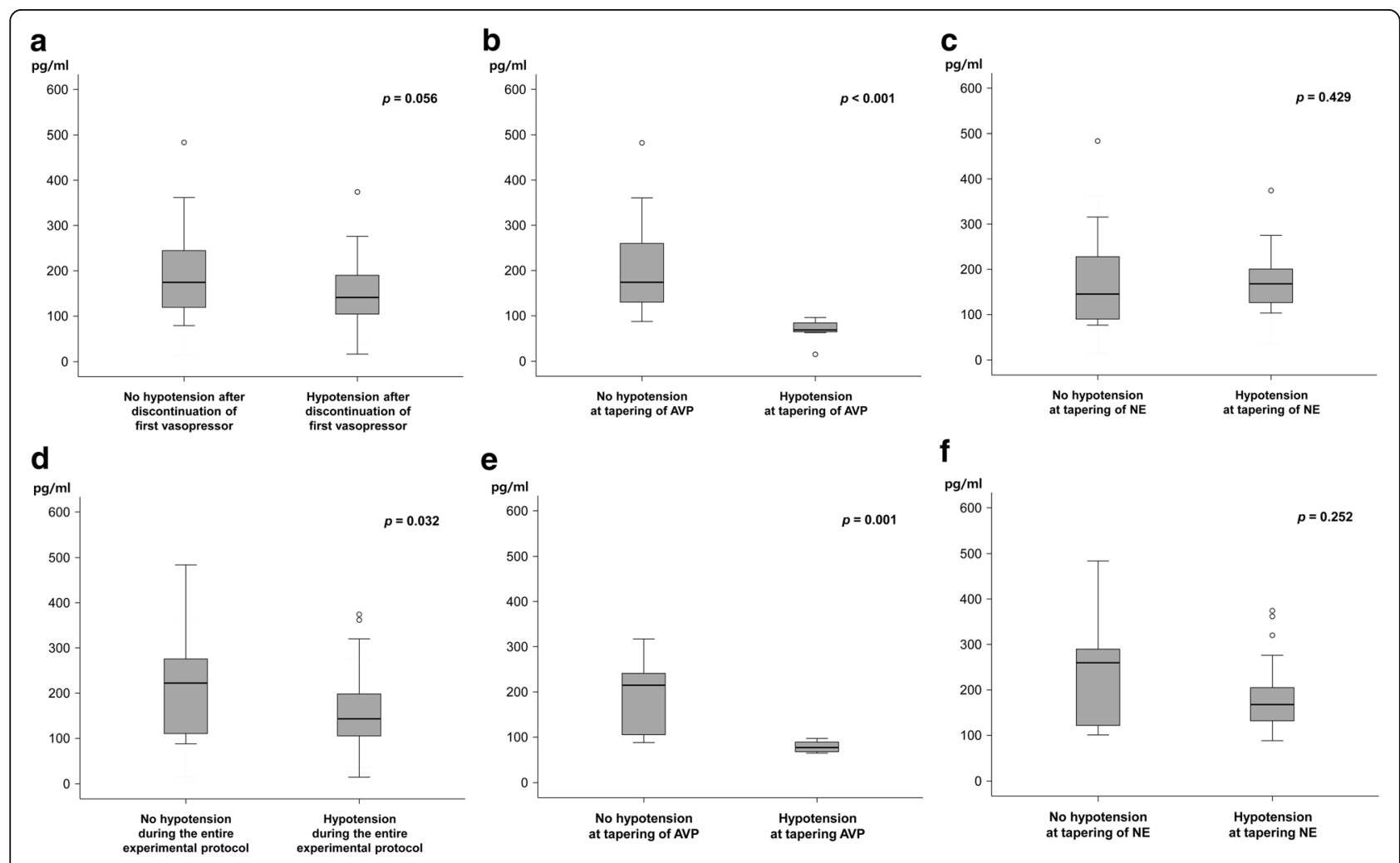

Fig. 3 Comparisons of serum copeptin levels between patients with and without hypotension after the first vasopressor was tapered (a-c) and after sequential tapering all infused vasopressors (d-f). Data are expressed as medians (interquartile ranges). AVP, vasopressin; NE, norepinephrine 
Table 3 Univariable comparisons of clinical characteristics in patients with hypotension according to the vasopressor tapered immediately before developing hypotension

\begin{tabular}{|c|c|c|c|}
\hline Variables & $\begin{array}{l}\text { Hypotension on tapering } \\
\text { of AVP }(n=12)\end{array}$ & $\begin{array}{l}\text { Hypotension on tapering } \\
\text { of NE }(n=46)\end{array}$ & $P$ value \\
\hline Age & $67(59-69)$ & $65(56-73)$ & 0.773 \\
\hline Gender, male & $10(83.3)$ & $30(65.2)$ & 0.307 \\
\hline Causes of septic shock ${ }^{a}$ & & & 0.246 \\
\hline Pneumonia & $8(66.7)$ & $20(43.5)$ & \\
\hline Intraabdominal infection & $2(16.7)$ & $14(30.4)$ & \\
\hline Urogenital infection & $3(25.0)$ & $6(13.0)$ & \\
\hline Cather related infection & $0(0.0)$ & $4(8.7)$ & \\
\hline Endocarditis & $0(0.0)$ & $2(4.3)$ & \\
\hline Others $^{b}$ & $0(0.0)$ & $3(6.5)$ & \\
\hline MAP before initial resuscitation, $\mathrm{mmHg}$ & $53(47-57)$ & $51(42-57)$ & 0.214 \\
\hline CVP before initial resuscitation, $\mathrm{mmHg}$ & $7(3-10)$ & $6(6-8)$ & 0.161 \\
\hline MAP at the time of vasopressor initiated, $\mathrm{mmHg}$ & $55(47-57)$ & $55(52-60)$ & 0.612 \\
\hline CVP at the time of vasopressor initiated, $\mathrm{mmHg}$ & $11(10-13)$ & $11(8-14)$ & 0.669 \\
\hline Total bilirubin, mg/dL & $0.90(0.35-3.05)$ & $1.20(0.50-2.70)$ & 0.382 \\
\hline Serum creatinine, mg/dL & $1.47(0.71-2.01)$ & $1.42(0.97-2.32)$ & 0.687 \\
\hline Lactic acid, $\mathrm{mmol} / \mathrm{L}(n=57)$ & $3.27(1.91-4.62)$ & $4.31(2.67-6.32)$ & 0.229 \\
\hline Procalcitonin, ng/mL $(n=53)$ & $13.09(4.18-81.53)$ & $11.01(2.61-30.97)$ & 0.602 \\
\hline C-reactive protein, $\mathrm{mg} / \mathrm{mL}$ & $14.87(8.51-29.72)$ & $12.57(5.59-19.42)$ & 0.129 \\
\hline Maximum NE dose during study period, ug/kg/min & $0.74(0.54-1.56)$ & $0.70(0.46-1.20)$ & 0.448 \\
\hline SAPS3 & $74(62-86)$ & $73(62-84)$ & 0.931 \\
\hline SOFA & $9(8-13)$ & $10(8-12)$ & 0.601 \\
\hline \multicolumn{4}{|l|}{ Clinical status on randomization } \\
\hline MAP, $\mathrm{mmHg}$ & $72(70-78)$ & $77(73-81)$ & 0.138 \\
\hline $\mathrm{CVP}, \mathrm{mmHg}$ & $10(8-12)$ & $10(8-14)$ & 0.420 \\
\hline Need for mechanical ventilation & $10(83.3)$ & $31(67.4)$ & 0.478 \\
\hline Need for renal replacement therapy & $4(33.3)$ & $14(30.4)$ & 1.000 \\
\hline Need for dobutamine & $1(8.3)$ & $3(6.5)$ & 1.000 \\
\hline SOFA & $13(11-16)$ & $12(9-15)$ & 0.255 \\
\hline PF ratio & $118.8(81.9-177.9)$ & $186.9(105.4-278.0)$ & 0.110 \\
\hline Total bilirubin, mg/dL & $1.35(0.53-3.68)$ & $1.40(0.80-3.88)$ & 0.744 \\
\hline Serum creatinine, mg/dL & $1.25(0.60-1.99)$ & $1.26(0.81-2.21)$ & 0.508 \\
\hline Corticosteroid treatment & $10(90.9)$ & $42(95.5)$ & 0.495 \\
\hline Copeptin, pg/mL $(n=56)$ & $77(67-90)$ & $168(131-207)$ & $<0.001$ \\
\hline Time to randomization, hours & $25.1(14.1-41.8)$ & $21.1(11.8-46.2)$ & 0.818 \\
\hline Time to discontinuation of vasopressors just before hypotension developed & $28.8(18.5-44.3)$ & $22.0(13.6-47.2)$ & 0.946 \\
\hline Time to hypotension after discontinuation of vasopressor, hours & $2.5(1.1-3.3)$ & $2.5(1.9-4.7)$ & 0.442 \\
\hline MAP at the time of hypotension developed on tapering of vasopressor, $\mathrm{mmHg}$ & $61(57-63)$ & $62(59-63)$ & 0.214 \\
\hline CVP at the time of hypotension developed on tapering of vasopressor, $\mathrm{mmHg}$ & $10(6-12)$ & $10(7-14)$ & 0.735 \\
\hline Total vasopressor duration, hours & $63.4(38.9-122.6)$ & $57.8(38.9-88.0)$ & 0.578 \\
\hline \multicolumn{4}{|l|}{ Clinical outcomes } \\
\hline ICU mortality & $5(41.7)$ & $13(28.3)$ & 0.486 \\
\hline ICU length of stay, days & $12(8-22)$ & $8(3-12)$ & 0.108 \\
\hline 28-day mortality & $5(41.7)$ & 17 (37.8) & 1.000 \\
\hline
\end{tabular}


Table 3 Univariable comparisons of clinical characteristics in patients with hypotension according to the vasopressor tapered immediately before developing hypotension (Continued)

\begin{tabular}{|c|c|c|c|}
\hline Variables & $\begin{array}{l}\text { Hypotension on tapering } \\
\text { of } \operatorname{AVP}(n=12)\end{array}$ & $\begin{array}{l}\text { Hypotension on tapering } \\
\text { of NE }(n=46)\end{array}$ & $P$ value \\
\hline Hospital mortality & $6(50.0)$ & $20(46.5)$ & 0.686 \\
\hline Hospital stay, days & $27(19-30)$ & $22(14-39)$ & 0.617 \\
\hline
\end{tabular}

Data are presented as frequencies (number of patients), with the percentage in parenthesis, or as medians with interquartile ranges (IQR) in parenthesis

${ }^{a}$ More than one criterion can be used

${ }^{b}$ Others included meningitis $(n=1)$ and deep neck $(n=1)$ and soft tissue infections $(n=1)$

$A V P$, vasopressin; CVP, central venous pressure; MAP, mean arterial pressure; NE, norepinephrine; PF ratio, arterial partial pressure of oxygen (PaO2)/fraction of inspired oxygen ( $\mathrm{FiO} 2$ ) ratio; SAPS3, simplified acute physiology score 3; SOFA, sequential organ failure assessment

[2]. However, there are no guidelines addressing the safe tapering of these medications in patients receiving AVP in addition to NE. Some physicians favor reducing NE first, because the incidence and duration of AVP deficiency is unclear due to variable causes of septic shock and disease courses $[22,23]$. In contrast, others suggest that AVP should be tapered first, because NE is easier to titrate than is AVP, as AVP significantly affects the cardiac output, splanchnic system, and balance between oxygen delivery and consumption [24]. In one retrospective cohort study, tapering of AVP before NE resulted in a greater incidence of clinically significant hypotension than did the reverse order [9]. In a more recent retrospective cohort study of 154 patients with septic shock, patients in whom AVP was tapered first developed hypotension that required intervention more commonly than did those in whom NE was tapered first [13]. In contrast to the findings from these retrospective observational studies $[9,13]$, however, the current prospective randomized controlled study revealed that NE tapering was more likely to lead to hypotension than AVP tapering during the entire experimental period $(79.3 \%$ vs $55.0 \%$, $p=0.035)$. It is difficult to explain this result; however, it might be explained by the different time to tapering vasopressors. In a prospective cohort study, AVP deficiency was mainly observed $\geq 36 \mathrm{~h}$ after shock onset [22]. In addition, interaction between AVP and corticosteroid treatment should be considered $[25,26]$. In the previous study [9] patients having NE discontinued first were more commonly treated with corticosteroids than those having AVP discontinued first. In this study, however, there was no difference in the dose and duration of corticosteroid infusion between the two groups. Difference in half-life between NE and AVP could affect our result. The longer effective half-life of AVP (10-20 min) than NE (2-2.5 min) may help avoid rebound hypotension after discontinuation of the drug.

Although multiple pathophysiologic mechanisms are responsible for cardiovascular failure in patients with septic shock [1], inadequate plasma concentrations of AVP prevent the restoration of normal vascular tone [1, 27]. Relative AVP deficiency has been reported in one third of patients with septic shock [22]. Theoretically, therefore, exogenous AVP administration could restore hemodynamic variables in septic shock that is poorly responsive to standard catecholamine therapies [28]. However, the exact onset time and frequency of AVP deficiency were not clearly determined $[22,23]$. Another question in this study sought to determine the association between AVP deficiency and the development of hypotension during AVP tapering. The incidence of hypotension during AVP tapering was $15 \%$ at the median time to AVP tapering of $29 \mathrm{~h}$. In addition, the serum levels of copeptin, the sensitive surrogate marker of AVP release [29], were significantly lower in these patients compared to those who did not develop hypotension. These results are comparable to those of previous studies, which indicated that the incidence of relative AVP deficiency was $15-22 \%$ approximately $24-36 \mathrm{~h}$ after shock onset $[22,23]$. However, the significant difference in serum copeptin was not observed in cases in which hypotension developed during NE tapering or in those without hypotension. Therefore, these finding suggest that the serum copeptin level is a useful surrogate marker to select patients who are more sensitive to exogenous AVP [30].

Our study has several limitations that should be mentioned. First, our study was conducted at a single center, which limits the generalization of our findings to other institutions or populations with different resources [31]. In the future, large multi-center trials could substantiate our findings. Second, patients in whom AVP was tapered first had lower PF ratios and therefore a greater need for mechanical ventilation, than did those in the NE group. This discrepancy may have been associated with more patients with pneumonia in the AVP group, which may have facilitated AVP secretion by hypoxemia [32], and could have an effect on our results. However, the serum copeptin level (directly mirroring AVP levels) at randomization was not significantly different between the two groups. Third, we did not have further information on myocardial dysfunction and its influence on our results. Therefore, further studies using advanced hemodynamic monitoring including cardiac index would be needed. Finally, this study was sufficiently powered to detect a difference in the primary outcome, but not clinically important secondary outcomes, such as mortality and length of stay in the ICU. 
Table 4 Univariable comparisons of clinical characteristics between patients with hypotension and without hypotension after sequential tapering all vasopressors

\begin{tabular}{|c|c|c|c|}
\hline Parameter & Hypotension $(n=58)$ & No hypotension $(n=20)$ & $P$ value \\
\hline Age & $65(57-72)$ & $66(54-70)$ & 0.828 \\
\hline Gender, male & $40(69.0)$ & $9(45.0)$ & 0.056 \\
\hline Causes of septic shock ${ }^{\mathrm{a}}$ & & & 0.588 \\
\hline Pneumonia & $28(48.3)$ & $11(55.0)$ & \\
\hline Intraabdominal infection & $16(27.6)$ & $8(40.0)$ & \\
\hline Urogenital infection & $9(15.5)$ & $5(25.0)$ & \\
\hline Cather related infection & $4(6.9)$ & $1(5.0)$ & \\
\hline Endocarditis & $2(3.4)$ & $0(0.0)$ & \\
\hline Others $^{b}$ & $3(5.2)$ & $0(0.0)$ & \\
\hline MAP before initial resuscitation, $\mathrm{mmHg}$ & $52(43-57)$ & $52(50-58)$ & 0.414 \\
\hline CVP before initial resuscitation, $\mathrm{mmHg}$ & $6(4-8)$ & $7(5-10)$ & 0.250 \\
\hline MAP at the time of vasopressor initiated, $\mathrm{mmHg}$ & $55(51-60)$ & $54(50-59)$ & 0.649 \\
\hline CVP at the time of vasopressor initiated, $\mathrm{mmHg}$ & $11(9-14)$ & $10(8-13)$ & 0.516 \\
\hline Total bilirubin, mg/dL & $1.15(0.50-2.70)$ & $0.90(0.60-1.83)$ & 0.406 \\
\hline Serum creatinine, mg/dL & $1.42(0.95-2.09)$ & $1.16(0.76-1.86)$ & 0.351 \\
\hline Lactic acid, mmol/L & $4.19(2.64-6.07)$ & $3.15(2.36-4.99)$ & 0.329 \\
\hline$P C T, n g / m L$ & $11.81(2.62-34.12)$ & $15.36(3.61-54.96)$ & 0.417 \\
\hline $\mathrm{CRP}, \mathrm{mg} / \mathrm{mL}$ & $12.60(5.83-21.19)$ & $20.66(11.97-26.10)$ & 0.026 \\
\hline Maximum NE dose during study period, ug/kg/min & $0.70(0.50-1.31)$ & $0.40(0.31-1.12)$ & 0.020 \\
\hline SAPS3 & $73(62-74)$ & $79(72-90)$ & 0.297 \\
\hline SOFA score & $10(8-12)$ & $10(8-11)$ & 0.936 \\
\hline \multicolumn{4}{|l|}{ Clinical status on randomization } \\
\hline MAP, $\mathrm{mmHg}$ & $77(71-80)$ & $76(68-83)$ & 0.936 \\
\hline $\mathrm{CVP}, \mathrm{mmHg}$ & $10(8-14)$ & $10(9-13)$ & 0.606 \\
\hline Need for mechanical ventilation & $41(70.7)$ & $13(65.0)$ & 0.635 \\
\hline Need for renal replacement therapy & $18(31.0)$ & $4(20.0)$ & 0.344 \\
\hline Need for dobutamine & $4(6.9)$ & $2(10.0)$ & 0.643 \\
\hline SOFA score & $12(10-15)$ & $12(9-15)$ & 0.704 \\
\hline PF ratio & $164.9(99.1-267.9)$ & $164.5(103.1-258.5)$ & 0.972 \\
\hline Total bilirubin, mg/dL & $1.40(0.78-3.70)$ & $1.25(0.50-3.73)$ & 0.453 \\
\hline Serum creatinine, mg/dL & $1.26(0.74-2.10)$ & $1.01(0.68-1.26)$ & 0.093 \\
\hline Corticosteroid treatment & $52(94.5)$ & $20(100.0)$ & 0.500 \\
\hline Copeptin, pg/mL $(n=76)$ & $144(105-199)$ & $223(109-281)$ & 0.032 \\
\hline Time to randomization, hours & $23.3(12.1-44.7)$ & $24.4(14.8-30.5)$ & 0.868 \\
\hline MAP at the evaluation of outcomes, $\mathrm{mmHg}$ & $62(59-63)$ & $72(67-77)$ & $<0.001$ \\
\hline CVP at the evaluation of outcomes, $\mathrm{mmHg}$ & $10(7-14)$ & $10(8-14)$ & 0.499 \\
\hline NE tapering at the evaluation of event outcomes & $46(79.3)$ & $11(55.0)$ & 0.035 \\
\hline AVP tapering at the evaluation of event outcomes & $12(20.7)$ & $9(45.0)$ & 0.035 \\
\hline Total vasopressor duration, hours & $57.8(39.0-110.6)$ & $30.8(21.3-36.7)$ & $<0.001$ \\
\hline \multicolumn{4}{|l|}{ Clinical outcomes } \\
\hline ICU mortality & $18(31.0)$ & $8(40.0)$ & 0.463 \\
\hline ICU length of stay, days & $9(4-13)$ & $7(3-12)$ & 0.387 \\
\hline 28-day mortality & $22(38.6)$ & $7(35.0)$ & 0.775 \\
\hline
\end{tabular}


Table 4 Univariable comparisons of clinical characteristics between patients with hypotension and without hypotension after sequential tapering all vasopressors (Continued)

\begin{tabular}{llll}
\hline Parameter & Hypotension $(n=58)$ & No hypotension $(n=20)$ & $P$ value \\
\hline Hospital mortality & $26(44.8)$ & $10(50.0)$ & 0.689 \\
Hospital length of stay, days & $23(14-37)$ & $19(14-42)$ & 0.936 \\
\hline
\end{tabular}

Data are presented as frequencies (number of patients), with the percentage in parenthesis, or as medians with interquartile ranges (IQR) in parenthesis ${ }^{a}$ More than one criterion can be used

${ }^{\mathrm{b} O t h e r s ~ i n c l u d e d ~ m e n i n g i t i s ~}(n=1)$ and deep neck $(n=1)$ and soft tissue infections $(n=1)$

AVP, vasopressin; CVP, central venous pressure; MAP, mean arterial pressure; NE, norepinephrine; PF ratio, arterial partial pressure of oxygen (PaO2)/fraction of inspired oxygen ( $\mathrm{FiO} 2$ ) ratio; SAPS3, simplified acute physiology score 3; SOFA, sequential organ failure assessment

\section{Conclusion}

The incidence of hypotension was high during vasopressor tapering, which was related to the vasopressor itself but not to the order of vasopressor tapering. Given the tentative results from our study, further studies with larger sample sizes are required to better determine the appropriate strategy for vasopressor tapering. However, NE tapering was significantly associated with hypotension developed during vasopressor tapering. Therefore, our results suggest that tapering AVP before NE (rather than the reverse) may lead to a lower incidence of hypotension in patients recovering from septic shock who are on concomitant AVP and NE.

\section{Abbreviations}

AVP: Vasopressin; CVP: Central venous pressure; EGDT: Early goal directed therapy; ICU: Intensive care unit; MAP: Mean arterial pressure; NE: Norepinephrine; PF ratio: Arterial partial pressure of oxygen ( $\mathrm{PaO} 2) /$ fraction of inspired oxygen (FiO2) ratio; SAPS3: Simplified acute physiology score 3; $\mathrm{SCvO}_{2}$ : Central venous oxygen saturation; SOFA: Sequential organ failure assessment

\section{Acknowledgements}

The authors thank Dr. So Yeon Lim for assistance in designing and initiating the research.

\section{Funding}

This study was supported by a grant from Samsung Medical Center (SMR112132).

\section{Availability of data and materials}

All data generated or analyzed during the present study are included in this published article.

\section{Authors' contributions}

Conception and design KJ, JUS, GYS; data analysis and interpretation: KJ, JUS, CRC, JHY, GYS; drafting the manuscript for intellectual content: KJ, JUS; revision of the manuscript: KJ, JUS, CRC, JHY, GYS. All authors read and approved the final manuscript.

\section{Ethics approval and consent to participate}

The institutional review board of the Samsung Medical Center approved this study and informed consent was obtained from each patient or the patient's relative or surrogate.

\section{Competing interests}

The authors declare that they have no competing interests.

\section{Publisher's Note}

Springer Nature remains neutral with regard to jurisdictional claims in published maps and institutional affiliations.

\section{Author details}

${ }^{1}$ Department of Critical Care Medicine, Samsung Medical Center, Sungkyunkwan University School of Medicine, Seoul, Republic of Korea. ${ }^{2}$ Division of Pulmonary and Critical Care Medicine, Department of Medicine, and Critical Care Medicine, Samsung Medical Center, Sungkyunkwan University School of Medicine, 81 Irwon-ro, Gangnam-gu, Seoul 06351, Republic of Korea. ${ }^{3}$ Division of Pulmonary and Critical Care Medicine, Department of Internal Medicine, Kangbuk Samsung Hospital, Sungkyunkwan University School of Medicine, Seoul, South Korea. ${ }^{4}$ Division of Cardiology, Department of Medicine, Samsung Medical Center, Sungkyunkwan University School of Medicine, Seoul, Republic of Korea.

Received: 14 December 2017 Accepted: 9 April 2018

Published online: 21 May 2018

\section{References}

1. Annane D, Bellissant E, Cavaillon JM. Septic shock. Lancet. 2005;365:63-78.

2. Rhodes A, Evans LE, Alhazzani W, Levy MM, Antonelli M, Ferrer R, Kumar A, Sevransky JE, Sprung CL, Nunnally ME, Rochwerg B, Rubenfeld GD, Angus DC, Annane D, Beale RJ, Bellinghan GJ, Bernard GR, Chiche JD, Coopersmith C, De Backer DP, French CJ, Fujishima S, Gerlach H, Hidalgo JL, Hollenberg SM, Jones AE, Karnad DR, Kleinpell RM, Koh Y, Lisboa TC, et al. Surviving Sepsis Campaign: international guidelines for management of sepsis and septic shock: 2016. Intensive Care Med. 2017;43:304-77.

3. Hollenberg SM. Vasoactive drugs in circulatory shock. Am J Respir Crit Care Med. 2011;183:847-55.

4. Gordon AC, Mason AJ, Thirunavukkarasu N, Perkins GD, Cecconi M, Cepkova M, Pogson DG, Aya HD, Anjum A, Frazier GJ, Santhakumaran S, Ashby D, Brett SJ, Investigators $V$. Effect of early vasopressin vs norepinephrine on kidney failure in patients with septic shock: the VANISH randomized clinical trial. JAMA. 2016; 316:509-18.

5. Vail EA, Gershengorn HB, Hua M, Walkey AJ, Wunsch H. Epidemiology of vasopressin use for adults with septic shock. Ann Am Thorac Soc. 2016;13: 1760-7.

6. Merouani M, Guignard B, Vincent F, Borron SW, Karoubi P, Fosse JP, Cohen $Y$, Clec'h C, Vicaut E, Marbeuf-Gueye C, Lapostolle F, Adnet F. Norepinephrine weaning in septic shock patients by closed loop control based on fuzzy logic. Crit Care. 2008;12:R155.

7. Persichini R, Silva S, Teboul JL, Jozwiak M, Chemla D, Richard C, Monnet X. Effects of norepinephrine on mean systemic pressure and venous return in human septic shock. Crit Care Med. 2012;40:3146-53.

8. Guinot PG, Bernard E, Levrard M, Dupont H, Lorne E. Dynamic arterial elastance predicts mean arterial pressure decrease associated with decreasing norepinephrine dosage in septic shock. Crit Care. 2015;19:14.

9. Bauer SR, Aloi JJ, Ahrens CL, Yeh JY, Culver DA, Reddy AJ. Discontinuation of vasopressin before norepinephrine increases the incidence of hypotension in patients recovering from septic shock: a retrospective cohort study. J Crit Care. 2010;25:362 e367-11.

10. Landry DW, Levin HR, Gallant EM, Ashton RC Jr, Seo S, D'Alessandro D, Oz MC, Oliver JA. Vasopressin deficiency contributes to the vasodilation of septic shock. Circulation. 1997;95:1122-5.

11. Tsuneyoshi I, Yamada H, Kakihana Y, Nakamura M, Nakano Y, Boyle WA 3rd. Hemodynamic and metabolic effects of low-dose vasopressin infusions in vasodilatory septic shock. Crit Care Med. 2001;29:487-93.

12. Rachoin JS, Dellinger RP. Timing of norepinephrine in septic patients: NOT too little too late. Crit Care. 2014;18:691.

13. Hammond DA, McCain K, Painter JT, Clem OA, Cullen J, Brotherton AL, Chopra D, Meena N. Discontinuation of vasopressin before norepinephrine 
in the recovery phase of septic shock. J Intensive Care Med. 2017: 885066617714209. https://doi.org/10.1177/0885066617714209. [Epub ahead of print].

14. Dellinger RP, Carlet JM, Masur H, Gerlach H, Calandra T, Cohen J, GeaBanacloche J, Keh D, Marshall JC, Parker MM, Ramsay G, Zimmerman JL, Vincent JL, Levy MM, Surviving Sepsis Campaign Management Guidelines C. Surviving Sepsis Campaign guidelines for management of severe sepsis and septic shock. Crit Care Med. 2004;32:858-73.

15. Jeon K, Shin TG, Sim MS, Suh GY, Lim SY, Song HG, Jo IJ. Improvements in compliance with resuscitation bundles and achievement of end points after an educational program on the management of severe sepsis and septic shock. Shock. 2012;37:463-7.

16. Rivers E, Nguyen B, Havstad S, Ressler J, Muzzin A, Knoblich B, Peterson E, Tomlanovich M, Early Goal-Directed Therapy Collaborative G. Early goaldirected therapy in the treatment of severe sepsis and septic shock. N Engl J Med. 2001;345:1368-77.

17. Annane D, Sebille V, Charpentier C, Bollaert PE, Francois B, Korach JM, Capellier G, Cohen Y, Azoulay E, Troche G, Chaumet-Riffaud P, Bellissant E. Effect of treatment with low doses of hydrocortisone and fludrocortisone on mortality in patients with septic shock. JAMA. 2002;288:862-71.

18. Robertson GL, Mahr EA, Athar S, Sinha T. Development and clinical application of a new method for the radioimmunoassay of arginine vasopressin in human plasma. J Clin Invest. 1973;52:2340-52.

19. Morgenthaler NG, Struck J, Alonso C, Bergmann A. Assay for the measurement of copeptin, a stable peptide derived from the precursor of vasopressin. Clin Chem. 2006:52:112-9.

20. Jochberger S, Luckner G, Mayr VD, Wenzel V, Morgenthaler NG, Friesenecker $\mathrm{BE}$, Hasibeder WR, Dunser MW. Course of vasopressin and copeptin plasma concentrations in a patient with severe septic shock. Anaesth Intensive Care. 2006;34:498-500.

21. Lan KG, DeMets DL. Discrete sequential boundaries for clinical trials. Biometrika. 1983;70:659-63.

22. Sharshar T, Blanchard A, Paillard M, Raphael JC, Gajdos P, Annane D. Circulating vasopressin levels in septic shock. Crit Care Med. 2003;31:1752-8.

23. Jochberger S, Mayr VD, Luckner G, Wenzel V, Ulmer H, Schmid S, Knotzer H, Pajk W, Hasibeder W, Friesenecker B, Mayr AJ, Dunser MW. Serum vasopressin concentrations in critically ill patients. Crit Care Med. 2006;34:293-9.

24. Klinzing S, Simon M, Reinhart K, Bredle DL, Meier-Hellmann A. High-dose vasopressin is not superior to norepinephrine in septic shock. Crit Care Med. 2003;31:2646-50

25. Russell JA, Walley KR, Gordon AC, Cooper DJ, Hebert PC, Singer J, Holmes CL, Mehta S, Granton JT, Storms MM, Cook DJ, Presneill JJ, Dieter Ayers for the V, Septic Shock Trial I. Interaction of vasopressin infusion, corticosteroid treatment, and mortality of septic shock. Crit Care Med. 2009;37:811-8.

26. Gordon AC, Mason AJ, Perkins GD, Stotz M, Terblanche M, Ashby D, Brett SJ. The interaction of vasopressin and corticosteroids in septic shock: a pilot randomized controlled trial. Crit Care Med. 2014;42:1325-33.

27. Landry DW, Oliver JA. The pathogenesis of vasodilatory shock. N Engl J Med. 2001;345:588-95.

28. Mutlu GM, Factor P. Role of vasopressin in the management of septic shock. Intensive Care Med. 2004;30:1276-91.

29. Palmiere C, Augsburger M. Copeptin as a diagnostic biomarker for sepsisrelated deaths. Peptides. 2014;59:75-8.

30. Jochberger S, Dorler J, Luckner G, Mayr VD, Wenzel V, Ulmer H, Morgenthaler NG, Hasibeder WR, Dunser MW. The vasopressin and copeptin response to infection, severe sepsis, and septic shock. Crit Care Med. 2009; 37:476-82.

31. Bellomo R, Warrillow SJ, Reade MC. Why we should be wary of single-center trials. Crit Care Med. 2009:37:3114-9.

32. Holmes CL, Patel BM, Russell JA, Walley KR. Physiology of vasopressin relevant to management of septic shock. Chest. 2001:120:989-1002.

Ready to submit your research? Choose BMC and benefit from:

- fast, convenient online submission

- thorough peer review by experienced researchers in your field

- rapid publication on acceptance

- support for research data, including large and complex data types

- gold Open Access which fosters wider collaboration and increased citations

- maximum visibility for your research: over $100 \mathrm{M}$ website views per year

At BMC, research is always in progress.

Learn more biomedcentral.com/submissions 